\title{
Note on the Oxidisable Organic Matter of Sea Water
}

\author{
By \\ W. R. G. Atkins, O.B.E., Sc.D., F.I.C., \\ Head of the Department of General Physiology at the Plymouth Laboratory.
}

In a former paper (1922) it was shown that the $\mathrm{pH}$ value of stored sea water decreased owing to the production of carbonic acid by organisms in it, and the oxidisable matter as indicated by this biological method appeared to be higher than the result obtained by oxidation with permanganate, according to various analyses available for comparison. These, however, were not done on the same water, so an attempt was made to determine this quantity on water of the English Channel off Plymouth. As is well known the permanganate method gives results which vary appreciably, according to the conditions of the experiment.

The standard method of the Public Health Association of the U.S.A. was adopted, with a slight modification necessary for its use with sea water. The solutions required are as follows :-

1. One volume of pure sulphuric acid diluted with three of distilled water. To this dilute potassium permanganate is added till a faint pink persists after standing for several hours.

2. Pure dry sodium oxalate, $0 \cdot 959 \mathrm{grm}$. per litre, giving 1 c.c. equivalent to 0.1 mgrm. of oxygen; the solution was preserved with toluene. (The standard method is to take $0.888 \mathrm{grm}$. of ammonium oxalate, without preservative.)

3. Permanganate solution, $0 \cdot 4 \mathrm{grm}$. per litre. This was allowed to stand in a warm place for over a fortnight to oxidise traces of organic matter. A more stable solution is thus obtained. To standardise the permanganate the procedure is as follows:-

10 c.c. sulphuric acid, 10 c.c. permanganate and 100 c.c. of freshly distilled water are placed on a boiling water bath for thirty minutes in 
, a chemically clean, conical flask of hard glass; 10 c.c. of sodium oxalate are then added, followed by permanganate till a faint pink persists in the hot liquid for several minutes. This treatment destroys the oxygenconsuming capacity of the water. After adding 10 c.c. of sodium oxalate, permanganate is run in till a faint excess persists ; duplicate determinations gave 11.47 and 11.49 c.c. of permanganate, and the latter value was adopted, no attempt being made to adjust exactly to $10 \cdot 0$ c.c., since it is necessary to restandardise at intervals. The above permanganate solution is accordingly equivalent to $0.087 \mathrm{mgrms}$. of oxygen per c.c.

When the water contains appreciable quantities of chloride, as in some sewage effluents, the official method is to digest in alkaline solution, instead of in acid, to avoid evolution of chlorine. For this purpose 10 c.c. of permanganate is added to 100 c.c. of the sample, followed by 0.5 c.c. 50 per cent sodium hydroxide. After digestion as before for thirty minutes 5 c.c. of sulphuric acid and 10 c.c. of oxalate are added, and the latter is titrated back with permanganate. Plymouth tap water tested by acid and alkaline oxidation was found to absorb 1.65 and 1.63 milligrams of oxygen per litre respectively, figures which may be considered identical. The newly distilled water took $\% \cdot 64$ mgrms., and a fresh-water pond fairly rich in algæ absorbed $3 \cdot 34$ mgrms.

Considerable difficulty was experienced in applying either method to sea water, and a number of determinations had to be rejected. It is impossible to use the acid oxidation on account of evolution of chlorine. With the alkaline method, as laid down, 5 c.c. of sulphuric acid is added, followed by the standard oxalate to destroy permanganate, excess being titrated back as before. This gives consistent results even when the chloride is as high as one per thousand, viz. 5 c.c. of sea water made up to 100 c.c. with distilled water, but the method is then of insufficient delicacy to detect with certainty the small differences met with in sea water from place to place. With undiluted sea water it is however unreliable, owing to evolution of chlorine, which is readily detected by its smell.

An attempt was made to omit the addition of acid, but in alkaline solution the reaction between permanganate and oxalate proceeds with surprising slowness even at boiling point. Consistent results may, however, be obtained as follows. After digestion 10 c.c. of the oxalate are added immediately to the alkaline solution and the acid is added in small portions while the liquid is kept in rapid rotation; when the colour has disappeared the permanganate from the burette is added with all speed to the moving liquid, and if overshot a duplicate may be done. The end point taken is the persistence of the pink for about a minute. 
Estimations carried out as described gave the results shown below :Station E1, 10 m. S.W. of Eddystone, taken $12 / 3$, stored 7 days . $\quad 1.00$ Same sample, filtered through Doulton candle . . . . 2.37 Near Eddystone, taken $7 / 3$, stored 12 days, filtered through Doulton candle . . . . . . . . . . 1.97

Same sample, stored 25 days, cleared with a few drops of ferric chloride and filter paper . . . . . . . . . . . . 0.76 Near Eddystone, taken 8/3, stored 11 days _ . . . . 0.84 " $\quad$ " 21 days $\cdot . \dot{0} \cdot 1.08$ hour at $100^{\circ}$ C. with 10 c.c. $\mathrm{N} / 10 \mathrm{H}_{2} \mathrm{SO}_{4}$ per 100 c.c. sea water . $\quad 2 \cdot 12$ Culture of Nitzschia closterium, 510,000 per c.c. . . . . . . . $3 \cdot 10$ Ditto, but digested for 80 minutes instead of $30 \quad$. $\quad . \quad 4.78$

The values obtained with untreated sea water after half an hour's digestion are approximately the final values, as prolongation to one hour only resulted in an extra $0 \cdot 1$ c.c. of permanganate being absorbed. It is clear that much of the organic matter exists in a form in which it is not readily oxidised by permanaganate, since higher values are obtained on storing and after hydrolysis; also it is obvious that the water at E1 in March cannot contain anything approaching 100,000 diatoms per c.c., as one would infer were the organic matter measurable by the oxygen absorption.

No method has as yet been used for estimating the oxygen absorption apart from the suspended organic matter, including living organisms, but it is hoped that this may be done by means of the Sharples super-centrifuge. The figures given show that the Doulton filter candle, after a considerable amount of washing, contaminated the water, but treatment with ferric chloride, which gives a precipitate in sea water, appears worth further trial.

\section{SUMMARY.}

1. Oxidation with alkaline permanganate and titration, according to the method of the American Public Health Association, can be used to estimate the oxygen absorption of water containing one part of chloride per thousand, but not of sea water.

2. By adding the oxalate solution to the hot alkaline permanganate after digestion and then acidifying cautiously, with the liquid in rapid rotation, it is possible to work with undiluted sea water if the final titration be also made with all speed. 
3. The water of the English Channel in March absorbs about one milligramme of oxygen per litre, the amount being increased somewhat by storing the water, also by hydrolysis of the algal plankton with dilute acid.

\section{REFERENCES.}

American Public Health Association. Standard Methods for the Examination of Water and Sewage. Boston, 1920.

Atkıns, W. R. G. The Respirable Organic Matter of Sea Water. This Journal, 1922, 12, 772-80. 